\title{
Evaluation of therapeutic effect of transcutaneous electrical acupoint stimulation on bone metastasis pain and its influence on immune function of patients
}

\author{
Jia-Bin Tai ${ }^{1}$, Liang Hong ${ }^{1}$, Meng-En $\mathrm{Ma}^{1}$, Jia Xu ${ }^{1}$, Jian-Qiao Fang ${ }^{2}$, Yi-Qian Jiang ${ }^{1}$ \\ ${ }^{1}$ Internal Medicine-Oncology, Xiaoshan First Hospital Affiliated to Medical College of Hangzhou Normal University, Hangzhou, China; ${ }^{2}$ Zhejiang \\ University of Traditional Chinese Medicine, Hangzhou, China \\ Contributions: (I) Conception and design: JB Tai, L Hong, ME Ma; (II) Administrative support: J Xu, JQ Fang; (III) Provision of study materials or \\ patients: JQ Fang, YQ Jiang; (IV) Collection and assembly of data: JB Tai, YQ Jiang; (V) Data analysis and interpretation: L Hong, ME Ma, J Xu; (VI) \\ Manuscript writing: All authors; (VII) Final approval of manuscript: All authors. \\ Correspondence to: Yi-Qian Jiang, PhD. Internal Medicine-Oncology, Xiaoshan First Hospital Affiliated to Medical College of Hangzhou Normal \\ University, No. 199 Shixin South Road, Xiaoshan District, Hangzhou 311200, China. Email: yiqian9988@163.com.
}

Background: This study aimed to observe the effect of transcutaneous electrical acupoint stimulation (TEAS) on bone metastasis pain, anxiety and immune function in cancer patients.

Methods: A total of 127 patients with bone metastasis pain from malignant tumors were treated with TEAS or drugs. The TEAS group comprised of 62 patients, while the drug control group comprised of 65 patients. The differences in general indexes, baseline pain and anxiety between these two groups were not statistically significant.

Results: Compared with those before treatment, the visual analog scale (VAS) scores of patients in the TEAS group and drug control group decreased after treatment, and the differences were statistically significant. The degrees of pain relief after treatment were similar between the two groups. Anxiety improved in both the TEAS group and drug control group, and the difference between these two groups was not statistically significant. The differences in immune indexes, B cell and CD8 between the TEAS group and drug control group were statistically significant. TEAS treatment could improve the declining trend of CD8 in patients.

Conclusions: TEAS treatment and drug treatment can effectively alleviate mild to moderate pain in patients, and the efficacy of these two groups is similar. The treatment for moderate and severe pain needs to be combined with drug treatment to achieve a control effect. TEAS can improve the anxiety and immune function of patients, and prevent the decrease in B cells and CD8 in patients treated with drugs alone.

Keywords: Transcutaneous acupoint electrical stimulation; cancerous pain; tumor; immunity; clinical research

Submitted Oct 31, 2019. Accepted for publication Aug 05, 2020.

doi: 10.21037/apm-19-434

View this article at: http://dx.doi.org/10.21037/apm-19-434

\section{Introduction}

Cancer pain remains as the focus and a challenge in cancer treatment. Opioids are the best choice for chronic moderate to severe cancer pain, but these are not omnipotent. Some patients suffer from bone metastasis pain due to invasion of the bone or periosteum by tumors. Hence, these patients need non-steroidal drugs, antidepressants, convulsants, and even nerve ablation. Meanwhile, patients have unavoidable side effects, such as constipation, vomiting and dizziness. With the increase in dosage, the side effects further increase, which limit the application of these drugs.

Transcutaneous electrical acupoint stimulation (TEAS) is a peripheral stimulation. It is an electrotherapy method for relieving pain by delivering low-frequency pulsed 
current to the human body through the skin. Electrodes are mostly placed on specific acupoints, which can produce acupuncture-like effects. Frequency conversion and different current intensity are commonly used in clinical application, in order to make the TEAS produce a variety of stimuli, inducing the central nervous system to secrete enough analgesic substances to achieve better therapeutic effects (1-8). Preliminary studies have been carried out to explore this mechanism (5,9-13). However, most of these clinical trials are not randomized enough, and there was no control group. Hence, these are far from the present requirements of evidence-based medicine $(14,15)$. Therefore, the present study was designed based on the unsatisfactory therapeutic effect of bone metastasis cancer pain treatment, with the aim of improving the therapeutic effect of bone metastasis cancer pain by taking advantage of the skin-sticking electro-acupuncture, which has less side effects and good tolerance. Furthermore, this approach also reduces the dosage and side effects of opioids, such as morphine, and improves the quality of life of patients. We present the following article/case in accordance with the CONSORT Reporting Checklist (available at http://dx.doi. org/10.21037/apm-19-434).

\section{Methods}

\section{Patient source}

The present study was carried out in accordance with the Declaration of Helsinki (as revised in 2013) and the relevant standards and regulations for clinical trials in China. The Ethics Committee approved the present study prior to its implementation (No. 2015-001). All patients provided a signed informed consent. A total of 130 patients with common bone metastases, and long-term persistent pain or recurrent paroxysmal pain, who could be treated with TEAS during hospitalization from January 2015 to December 2017, were enrolled into the present study. Registration number is ChiCTR2000030549 and name of trial register is Chinese Clinical Trial Registry).

\section{Research methods}

The randomized control method was adopted (SPSS software randomized grouping) to divided these patients into the following two groups.

TEAS group $(n=65)$ : Han's six-lead TEAS machine (HANS-100B) was used for TEAS for 5 days, twice a day.
Acupoint selection: double upper limb: bilateral Hegu + Neiguan/Waiguan (Waiguan was selected for patients with upper limb pain); both lower limbs: Zusanli + Sanyinjiao were selected; Jiaji acupoints on both sides of the target site (on both sides of the spinous process, 0.5 cuns apart). The $2 / 100 \mathrm{~Hz}$ dilatational wave was adopted. The present intensity was appropriate when the patient's maximum tolerance was reached. The range was within $10-15 \mathrm{~mA}$ for the upper limb, the range was within $15-30 \mathrm{~mA}$ for the lower limb, and the range was $25-40 \mathrm{~mA}$ for the Jiaji acupoint area.

Drug control group ( $n=62)$ : drugs + false TEAS (the selected acupoints were the same with those in the TEAS treatment group, the current was set to $1 \mathrm{~mA}$ ) was used for the treatment. According to the National Comprehensive Cancer Network (NCCN) 2018 edition of the Guidelines for Adult Cancer Pain, the dosimetric titration and adjustment of opioids, and conversion of equivalent dosage of opioids were carried out. These drugs were continuously administered for 5 days according to the design of the study.

In the present study, TEAS treatment was singleblinded (patient). Since the present study used a singleblinded control method, each patient was $50 \%$ likely to enter the control group. Therefore, from the $6^{\text {th }}$ day of the trial, patients with poor efficacy in the two groups were given the corresponding drug treatment or TEAS treatment, according to the patient's preference. Patients in the TEAS group and drug control group were required to fill in the case report form, "A Prospective Clinical Study (XSFHCC-001) on the Observation of the Curative Effect of Electro-Acupuncture on Bone Metastasis Pain in Cancer Patients", before treatment, in order to complete the anxiety survey. The visual analog scale (VAS) scores were assessed at the beginning and end of the trial, and before and after each treatment.

\section{Observation indexes}

\section{Pain intensity assessment}

VAS score: 0 indicates painless, 10 indicates severe pain, and scores between the two ends part indicate varying degrees of pain.

\section{Degree of anxiety and pain}

The Self-Rating Anxiety Scale (SAS) was used to evaluate the patients. The criteria are as follows: (I) no presence or presence for a very short time; (II) presence sometimes; (III) presence most of the time; 4 , presence most of the time. 
Table 1 VAS scores before and after treatment (mean \pm SD)

\begin{tabular}{lccccc}
\hline Groups & $\mathrm{N}$ & Mild & Moderate & Score before first treatment & Score after last treatment \\
\hline TEAS group & 62 & 41 & 21 & $3.74 \pm 1.17$ & $1.19 \pm 0.99$ \\
Drug control group & 65 & 38 & 27 & $4.00 \pm 2.26$ & $1.44 \pm 1.02$ \\
P & - & - & - & 0.418 & 0.182 \\
\hline
\end{tabular}

VAS, visual analog scale; SD, standard deviation; TEAS, transcutaneous electrical acupoint stimulation.

The curative effect was evaluated according to the pain grading method (VAS score): complete remission (CR), no pain after treatment; partial remission (PR), pain after treatment is significantly milder than that before administration, sleep is basically undisturbed, and the patient can live a normal life; mild remission (MR), the pain after treatment is milder than that before administration, but the patient still feels obvious pain, and sleep is still disturbed; non-remission (NR), there is no relief of pain after treatment, when compared to that before treatment. Effective remission rate $=(C R+P R) /$ case number $\times 100 \%$.

\section{Immune indicators}

Flow cytometry was used to detect T cells, B cells, $\mathrm{CD}^{+}{ }^{+} \mathrm{CD} 4^{+} \mathrm{T}$ cells, $\mathrm{CD}^{+} \mathrm{CD}^{+} \mathrm{T}$ cells and natural killing (NK) cells [D FACSC antoTM II Flow Cytometer (SN: V33947300234)]. A BD lymphocyte subgroup detection kit (batch number: 40902/95982/32954/31833/53789/70258/2 $2607 / 84752 / 40732$ ) was used.

\section{Result analysis}

Data were statistically analyzed using statistical software SPSS 20.0. All statistical tests were conducted using a onesided test. The significant level was set at $\mathrm{P} \leq 0.05$. Normally distributed measurement data were compared using $t$-test, while non-normally distributed data were compared using Wilcoxon rank-sum test. Count data were compared using Chi-square test.

\section{Results}

\section{General data}

The differences in age and gender between the two groups were not statistically significant. Furthermore, the differences in disease type and bone metastasis site were also not statistically significant.

\section{VAS scores before and after treatment}

All 127 patients had mild-to-moderate bone metastasis pain. The TEAS score was $3.74 \pm 1.17$ before the initial treatment and drug control group 4.00 \pm 2.26 before the initial treatment, the difference was not statistically significant. The scores in each group significantly decreased after the last treatment. The pairwise comparison using $t$-test revealed that the difference was statistically significant $(\mathrm{P}<0.01)$. After treatment, these scores were significantly lower in the TEAS group than in drug control group (Table 1).

\section{Anxiety degree}

In the TEAS group, the SAS score was 50.65 before treatment and 45.77 after treatment, and the difference was statistically significant $(\mathrm{P}=0.001)$. The SAS score for the TEAS group was 45.77 after treatment, while the SAS score for the drug control group was 47.78 after treatment, and the difference between these two groups was not statistically significant $(\mathrm{P}=0.160$, Table 2).

\section{Immune indexes}

Before treatment, the differences in B cell and CD8 between the two groups were statistically significant, but the differences in the remaining indexes were not statistically significant $(\mathrm{P}>0.05)$. After treatment, the differences in $\mathrm{B}$ cell and CD8 between the two groups were statistically significant, but the difference in the remaining indexes were not statistically significant (Table 3).

\section{Comparison of pain scores before and after treatment in the TEAS group}

In the present study, the VAS score for the TEAS group before and after 10 treatments were analyzed and summarized. The results revealed the following: first, 
Table 2 Comparison of anxiety scores of patients before and after treatment (mean \pm SD)

\begin{tabular}{lcccc}
\hline Groups & $\mathrm{N}$ & Sleep score before treatment & SAS score before treatment & SAS score after treatment \\
\hline TEAS group & 62 & $9.97 \pm 4.09$ & $50.65 \pm 8.01$ & $45.77 \pm 8.47$ \\
Drug control group & 65 & $10.00 \pm 5.36$ & $48.37 \pm 10.46$ & $47.78 \pm 7.59$ \\
$\mathrm{P}$ & - & 0.970 & 0.169 & 0.160 \\
\hline
\end{tabular}

SD, standard deviation; SAS, Self-Rating Anxiety Scale; TEAS, transcutaneous electrical acupoint stimulation.

Table 3 Comparison of the average number of immune cells in patients before and after treatment $(\mathrm{mean} \pm \mathrm{SD})$

\begin{tabular}{|c|c|c|c|c|c|c|c|}
\hline Groups & $\mathrm{N}$ & $\mathrm{T}$ & $\mathrm{B}$ & NK & CD4 & CD8 & CD4/CD8 \\
\hline TEAS group & 62 & $725.55 \pm 318.67$ & $162.19 \pm 116.50$ & $216.46 \pm 150.91$ & $420.00 \pm 266.21$ & $283.85 \pm 127.22$ & $1.71 \pm 1.34$ \\
\hline Drug control group & 65 & $874.95 \pm 272.49$ & $142.81 \pm 109.51$ & $229.83 \pm 191.29$ & $486.39 \pm 176.76$ & $372.60 \pm 162.27$ & $1.60 \pm 1.03$ \\
\hline $\mathrm{P}$ & & 0.005 & 0.336 & 0.664 & 0.102 & 0.001 & 0.623 \\
\hline TEAS group & 62 & $727.57 \pm 296.72$ & $172.69 \pm 140.22^{*}$ & $251.45 \pm 143.68$ & $437.71 \pm 246.87$ & $254.44 \pm 123.69^{\star}$ & $2.04 \pm 1.35$ \\
\hline Drug control group & 65 & $800.64 \pm 282.43$ & $127.32 \pm 105.56$ & $211.92 \pm 164.05$ & $467.81 \pm 180.91$ & $306.59 \pm 128.05$ & $1.81 \pm 1.06$ \\
\hline $\mathrm{P}$ & & 0.158 & 0.041 & 0.152 & 0.433 & 0.021 & 0.286 \\
\hline
\end{tabular}

*, $\mathrm{P}<0.05$. SD, standard deviation; TEAS, transcutaneous electrical acupoint stimulation.

after each TEAS treatment, the VAS score significantly decreased, when compared with that before treatment, and this increased again at the following day and repeatedly changed, presenting a serrated shape on the chart. Second, the comparison of scores before the first and $10^{\text {th }}$ treatment exhibited a gradually decreasing trend, and the difference was statistically significant. Finally, the comparison of scores after the first and $10^{\text {th }}$ treatment revealed a gradual decreasing trend, but the difference was not statistically significant (Figure 1).

\section{Overall efficacy evaluation}

In the drug control group, the total effective rate was $100 \%$, in which the cured rate was $24.6 \%$ and the improvement rate was $75.4 \%$. In the TEAS group, the total effective rate was $93.5 \%$, in which the cured rate was $24.2 \%$ and the improvement rate was $69.4 \%$. However, the difference between these two groups was not statistically significant (Table 4).

\section{Discussion}

In the present study, the exploration was carried out based on existing research. Focus was given on the aspect of bone metastatic cancer pain, which has special physiological and pathological characteristics, but is difficult to treat in clinic, in order to further alleviate the pain of patients and improve their quality of life.

Transcutaneous electrical nerve stimulation (TENS) is a peripheral stimulation that transmits low-frequency pulse current to the human body through the skin to relieve pain (16). It is different from traditional nerve stimulation in the following aspects: traditional electrical stimulation mainly stimulates motor fibers, while TENS mainly stimulates sensory fibers, and it can stimulate both sensory and motor fibers when the stimulus parameters are changed, or the stimulus reaches the threshold. TEAS has low frequency and high strength, and the electrodes are mostly placed on specific acupoints, which can produce similar effects, such as acupuncture and moxibustion. In clinic, frequency conversion and different current 


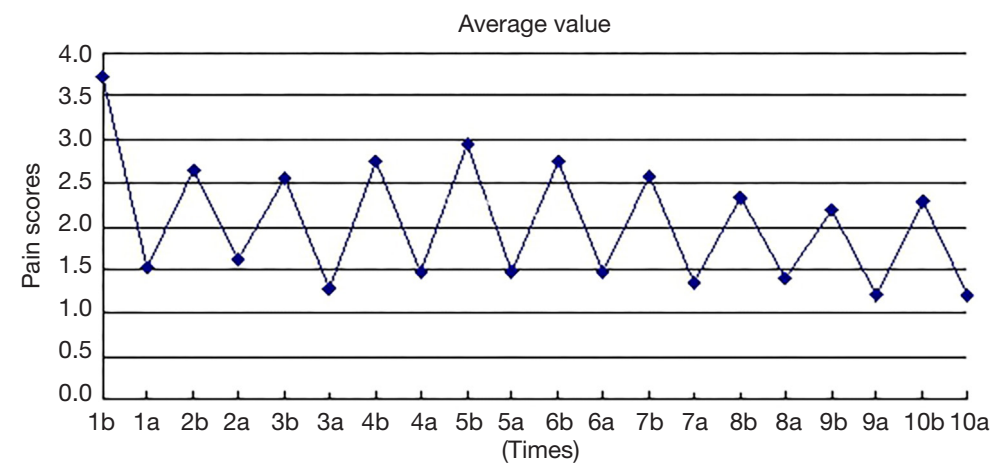

Figure 1 Distribution trend of average value pain scores. After each TEAS treatment, the VAS score significantly decreased, when compared with that before treatment, and this increased again at the following day and repeatedly changed, presenting a serrated shape on the chart. Second, the comparison of scores before the first and $10^{\text {th }}$ treatment exhibited a gradually decreasing trend, and the difference was statistically significant. Finally, the comparison of scores after the first and $10^{\text {th }}$ treatment revealed a gradual decreasing trend, but the difference was not statistically significant. Note: $\mathrm{a}=\mathrm{after} ; \mathrm{b}=$ before.

Table 4 Overall efficacy evaluation

\begin{tabular}{lcccc}
\hline Groups & CR & NR & PR & Total \\
\hline TEAS group & 15 & 4 & 43 & 62 \\
Drug control group & 16 & 0 & 49 & 65 \\
\hline
\end{tabular}

CR, complete remission; NR, non-remission; PR, partial remission.

intensities are commonly used to make TENS produce a variety of stimuli, causing the central nervous system to secrete enough analgesic substances to achieve a better therapeutic effect.

The present study revealed the following: (I) the pain score for the TEAS group exhibited a serrated shape on the chart before and after treatment, manifesting as a decrease in score after treatment, and after maintaining the analgesic effect for 6 hours, the pain score gradually increased and returned to the similar level before treatment; (II) the analgesic effect of TEAS was significant and persistent, especially at 5 days after treatment, and the VAS score exhibited a downward trend, when compared with that before treatment; (III) subgroup analysis: TEAS alone can relieve pain and has persistence; (IV) the total effective rate in the TEAS group was similar to that in the drug control group. These results reveal that acupuncture and moxibustion, or acupuncture and moxibustion combined with drugs can achieve the same analgesic effect as drugs. The results of the present study further confirm that acupuncture and moxibustion, or TEAS have significant analgesic effects, and that these analgesic effects were persistent. The reason may be the sustained release of endorphins stimulated by TEAS and the regulation of local inflammatory mediators. This needs to be further explored in combination with experimental research.

In terms of anxiety, the present study revealed the following: (I) the differences in SAS scores before and after treatment in the TEAS group was statistically significant; (II) the difference in SAS score between the TEAS group and drug control group was not statistically significant. These results reveal that acupuncture and moxibustion can alleviate the anxiety of patients with cancer pain, and achieve the same effect as drugs.

In terms of immune indexes, the present study revealed the following: (I) B cells: the difference between the two groups was statistically significant. (II) CD8: since the average value before treatment was higher in the drug control group than in the acupuncture group, the difference between these two groups was statistically significant, which cannot be compared with that after treatment. In order to further clarify the effect of acupuncture and moxibustion on CD8, this was compared before and after treatment in the TEAS group. The results revealed that the difference before and after treatment was not statistically significant. In the drug control group, this exhibited a significant downward trend after treatment. These results reveal that TEAS treatment can prevent the decrease in CD8 caused by drug therapy alone. However, the specific mechanism needs to be further clarified. (III) T, CD4, NK and CD4/ CD8: The differences between the TEAS group and drug control group were not statistically significant.

In the present study, although more evidence has been 
obtained to support TEAS analgesia, and confirmed its advantages of safety, low incidence of constipation, and ability to maintain the normalization of immune function, various detailed aspects still needs to be improved in the analgesic process. For example, pain must be assessed before the treatment. For patients with mild, moderate and severe pain, different treatment measures should be taken in time. Furthermore, the method of selecting acupoints should be unified as far as possible, and the acupoint selection method based on syndrome differentiation and local acupoints should be mainly applied (17). It is easy to identify the presence of needling sensation in patients with acupuncture or electro acupuncture experience. However, it is difficult to conduct a randomized, double-blinded, controlled trial on acupuncture and moxibustion, or electro-acupuncture in the treatment of cancer pain. It is recommended to use different current intensities as controls.

\section{Conclusions}

The analgesic effect of TEAS is significant and persistent. The mechanism may be correlated to the sustained release of endorphins stimulated by TEAS, the regulation of local inflammatory mediators, and the regulation of immune function. TEAS or TEAS combined with drugs can achieve the same analgesic effect as drugs, and can improve a patient's anxiety. Furthermore, TEAS can prevent the decrease in CD8 caused by drug therapy alone.

\section{Acknowledgments}

We are particularly grateful to all the people who have given us help on our article.

Funding: Hangzhou Science and technology project (No.20150633B53; No.20171226Y82) and Hangzhou Health Planning Project (No.2017B16).

\section{Footnote}

Reporting Checklist: The authors have completed the CONSORT Reporting Checklist. Available at http://dx.doi. org/10.21037/apm-19-434

Data Sharing Statement: Available at http://dx.doi. org/10.21037/apm-19-434

Conflicts of Interest: All authors have completed the ICMJE uniform disclosure form (available at http://dx.doi. org/10.21037/apm-19-434). The authors have no conflicts of interest to declare.

Ethical Statement: The authors are accountable for all aspects of the work in ensuring that questions related to the accuracy or integrity of any part of the work are appropriately investigated and resolved. I confirm that I have read the Editorial Policy pages. This study was conducted with approval from the Ethics Committee of Xiaoshan First Hospital Affiliated to Medical College of Hangzhou Normal University (No. 2015-001). This study was conducted in accordance with the declaration of Helsinki (as revised in 2013). Written informed consent was obtained from all participants.

Open Access Statement: This is an Open Access article distributed in accordance with the Creative Commons Attribution-NonCommercial-NoDerivs 4.0 International License (CC BY-NC-ND 4.0), which permits the noncommercial replication and distribution of the article with the strict proviso that no changes or edits are made and the original work is properly cited (including links to both the formal publication through the relevant DOI and the license). See: https://creativecommons.org/licenses/by-nc-nd/4.0/.

\section{References}

1. Zayan K, Aggarwal S, Felix E, et al. Transcutaneous Electrical Nerve Stimulation for the Long-Term Treatment of Ocular Pain. Neuromodulation 2020;23:871-7.

2. Wang JS, Yang J, Deng S, et al. Acupuncture combined with tamsulosin hydrochloride sustained-release capsule in the treatment of chronic prostatitis/chronic pelvic pain syndrome: A study protocol for a randomized controlled trial. Medicine (Baltimore) 2020;99:e19540.

3. Wu L, Yin Y, Sun K, et al. Effectiveness of acupuncture versus spinal-epidural anesthesia on labor pain: a randomized controlled trial. J Tradit Chin Med 2017;37:629-35.

4. Deng G, Bao T, Mao JJ. Understanding the Benefits of Acupuncture Treatment for Cancer Pain Management. Oncology (Williston Park) 2018;32:310-6.

5. He Y, Guo X, May BH, et al. Clinical Evidence for Association of Acupuncture and Acupressure With Improved Cancer Pain: A Systematic Review and MetaAnalysis. JAMA Oncol 2020;6:271-8.

6. Keilani M, Kainberger F, Pataraia A, et al. Typical aspects 
in the rehabilitation of cancer patients suffering from metastatic bone disease or multiple myeloma. Wien Klin Wochenschr 2019;131:567-75.

7. Sivanesan E, Gulati A. Resurgence of peripheral nerve stimulation with innovation in device technologies. Reg Anesth Pain Med 2019;44:615-6.

8. Zhang C, Xia C, Zhang X, et al. Wrist-ankle acupuncture attenuates cancer-induced bone pain by regulating descending pain-modulating system in a rat model. Chin Med 2020;15:13.

9. Lu W, Giobbie-Hurder A, Freedman RA, et al. Acupuncture for Chemotherapy-Induced Peripheral Neuropathy in Breast Cancer Survivors: A Randomized Controlled Pilot Trial. Oncologist 2020;25:310-8.

10. Deng G, Giralt S, Chung DJ, et al. Reduction of Opioid Use by Acupuncture in Patients Undergoing Hematopoietic Stem Cell Transplantation: Secondary Analysis of a Randomized, Sham-Controlled Trial. Pain Med 2020;21:636-42.

11. Menéndez-Aponte Y Guzmán RM, Turcott Chaparro JG, De la Piedra Gómez A, et al. Effect of complementary Integrative Oncology on anxiety, depression and quality of life in thoracic cancer patients: A pilot study. Complement
Ther Clin Pract 2019;36:56-63.

12. D'Alessandro EG, Nebuloni Nagy DR, de Brito CMM, et al. Acupuncture for chemotherapy-induced peripheral neuropathy: a randomised controlled pilot study. BMJ Support Palliat Care 2019. [Epub ahead of print]. doi:10.1136/bmjspcare-2018-001542.

13. Garland SN, Xie SX, DuHamel K, et al. Acupuncture Versus Cognitive Behavioral Therapy for Insomnia in Cancer Survivors: A Randomized Clinical Trial. J Natl Cancer Inst 2019;111:1323-31.

14. Li K, Giustini D, Seely D. A systematic review of acupuncture for chemotherapy-induced peripheral neuropathy. Curr Oncol 2019;26:e147-54.

15. Shi RY, Fu TF, Cai YQ, et al. Electroacupuncture intervention relieves pain possibly by promoting MOR endocytosis in locus coeruleus in bone cancer pain rats with morphine tolerance. Zhen Ci Yan Jiu 2019;44:161-9.

16. Kerai S, Saxena KN, Taneja B, Sehrawat L. Role of transcutaneous electrical nerve stimulation in postoperative analgesia. Indian J Anaesth 2014;58:388-93.

17. Kim TH, Kang JW. Acupuncture for symptoms management in Korean breast cancer survivors: a prospective pilot study. Acupunct Med 2019;37:164-74.
Cite this article as: Tai JB, Hong L, Ma ME, Xu J, Fang JQ, Jiang YQ. Evaluation of therapeutic effect of transcutaneous electrical acupoint stimulation on bone metastasis pain and its influence on immune function of patients. Ann Palliat Med 2020;9(5):2538-2544. doi: 10.21037/apm-19-434 